Dr. S. Weir Mitchell's fame as an original investigator and physiologist, already places him in the foremost rank; and we are confident that these contributions to the literature of a confessedly very difficult subject, cannot fail to add another leaf to his well earned laurels.

\title{
REVIEW III.
}

St. Bartholomew's Hospital Reports. Vol. III. pp. 486.

This volume of 'St. Bartholomew's Hospital Reports' contains twenty-five papers, and is prefaced by a memoir of the late Dr. Jeaffreson, physician to the hospital, who died at the end of the year 1866. The memoir does no more than justice to the memory of a most accomplished physician and estimable man, who, although he never lectured on medicine, and, unfortunately for his contemporaries and for posterity, never placed upon record the results of his extensive experience, was nevertheless held in the highest esteem by the students of the hospital, was consulted by a large circle of patients, and was beloved by a host of friends. It is satisfactory to learn that, although Dr. Jeaffreson had barely passed the middle period of life, his practice had been so successful that he amassed a very considerable fortune, and was about to retire, at least partially, from his professional pursuits, when death put an end to his distinguished career. Although his prospects, on starting in life, were not brilliant, he never had struggled with adversity : on the contrary, he was favoured in his youth by the friendship and active assistance of many persons of influence, and the advantages thus gained he turned to the very best account, and fully justified the estimate which had been formed of his character by those who were best qualified to form a judgment.

I. Some Statistics of Pyremia. By William S. SAvory, F.R.S.-In this paper Mr. Savory has collected the particulars of what seem to be the main features of 133 cases of pyæmia, ninety-five of them having been taken from the pages of the Medical Journals and the remaining thirty-eight having occurred in the wards of the hospital during three years. All the cases mentioned occurred in British practice, and no doubtful or questionable cases were included in the tables. Mr. Savory thinks that in some important facts the records of 
pyæmia are deficient, as, for instance, in omitting to mention whether there is any evidence of infection from without, and whether there has been proximity to other cases. Although pyæmia is often spoken of as arising from contagion, there is no evidence to show that it does so in the same manner as scarlatina or smallpox. But pyæmia commonly supervenes on foul wounds, and the emanations from these wounds may impregnate the atmosphere, and may be carried to adjacent healthy wounds in other persons, and in them create morbid action. It is generally thought that this disease is specially prevalent in hospitals, but this opinion may be partly due to the facts that patients are often admitted into hospitals who are already the victims of the disease, that cases in hospitals are more carefully recorded than those which occur in the houses of the poor, and that the great majority of persons who are the subjects of injuries or operations likely to be followed by pyæmia are admitted into hospitals. Still there can be no doubt that certain conditions sometimes prevailing in the best hospitals may predispose to pyæmia, because it is almost impracticable to avoid having an undue number of open wounds or sores in a ward, and then the atmosphere is likely to become tainted, in spite of the utmost care. The tables yield little support to the view that phlebitis or even thrombosis is a necessary antecedent of pyæmia. The earliest symptom of this disease, according to nearly all the tables, was rigor or shivering, and this symptom is the more diagnostic when, as is almost invariably the case in pyæmia, it is immediately or very rapidly followed by profuse sweating. The rigors appear to Mr. Savory to stamp the relationship of pyæmia to the specific fevers, and they may be regarded as indicating the operation of a poison in the blood in both cases.

\section{Practical Observations on the Nature and Medical Treat -} ment of Obstruction of the Bowels and upon Constipation. By Thomas Head, M.D.-Dr. Head draws a distinction between obstruction of the bowels, or an entire interruption of their ordinary functions, and constipation, which is a less serious affection. The former most commonly arises from intussusception and from the lodgment of particles of undigested food in or near the ileo-cæcal valve, and more rarely from hernia and from malignant disease of the bowels; the latter has its seat almost exclusively in the large intestine. In one of the cases related, which was that of a child five months old, there was obstinate obstruction of the bowels, and the treatment adopted by Dr. Head was to inject warm oil and quicksilver into the bowels, and then to hold the child up by the legs so as to allow 
the quicksilver to traverse the intestinal tube by gravitation. This practice, although novel, is described as being successful. In cases of malignant diseases nothing can be expected from treatment except the alleviation of the sufferings of the patient. Constipation of the bowels is a common affection, and it is almost incredible to what an extent the large intestines may be loaded with scybalous concretions without causing the patient any remarkable inconvenience. Married women, of middle age, have sometimes regarded the sense of fulness and weight of the abdomen as indicative of pregnancy. For the removal of such scybalous masses, the retention of which in the higher portions of the colon is likely sometimes to produce relaxation resembling diarrhœa, Dr. Head recommends small doses of blue pill and podophyllin, with a little acetate of lead, with about a quarter or a sixth of a grain of opium, and a drop of creosote at bedtime, for several successive nights, followed in the morning by a draught consisting of castor oil, liquor potassæ, a little chloroform, and a few drops of laudanum. Dr. Head notices that a laceration of the mucous membrane covering the sphincter ani is sometimes caused by the passage of hard scybalous fæces and by the forcible expulsive actions of the abdominal and intestinal muscles.

III. On Convulsions in Children. By SAmuel GeE, M.D. -In this paper, Dr. Gee gives a summary account of 102 cases of epileptiform convulsions in children, and he divides them into three classes according as their causes are local, general, or uncertain. In twenty-four cases the convulsions were apparently symptomatic of local disease in or near the cerebrum, such as tumours, abscesses, disease of the vertebræ, \&c. In 73 cases 1 was caused by anæmia, 1 by uræmia, 12 by acute specific diseases, as scarlet fever, measles, \&c.; 1 by syphilis, 1 by exhaustion from a large ulcer, and one from chronic Bright's disease, but 56 were unaccounted for, and they are classed under the head of convulsions depending upon the general condition of the child. Dr. Gee considers that many cases of essential convulsions (eclampsia) are associated with a constitution tending to rickets. After giving a brief abstract of the 56 cases, Dr. Gee remarks that hypertrophy of the brain sometimes co-exists with rickets; that sometimes enlargement of the cranial cavity occurs in rickets, without the brain being enlarged at the same time; that in reference to dentition, the backwardness of the teeth and the tendency to convulsions are sometimes concomitants of the ricketty diathesis, and that in several cases the occurrence of measles did not produce fits, although the children had been subject to them previously. The cases of convulsions of uncertain origin were only five in number. The 
treatment adopted in most of the cases consisted in the administration of the bromide of potassium or ammonium (for a child, for instance, of a year old), in doses of four grains three or four times a day, and when the fits had ceased, of cod-liver oil and vinum ferri.

IV. On the Relation of Life to other Forces. By W. Morrant Baker.-In this paper, which is altogether speculative and philosophical, Mr. Baker draws a comparison between the forces exercised under the influence of life and those which are displayed by inorganic matter. He shows that the actions of life consist in a great measure of utilising force, and making it subservient to special purposes, as when the heat of the sun under the influence of vegetation is made to assist in the decomposition of carbonic acid, and the formation of woody fibre. Vegetation exercises a chemical force in the evolution of oxygen, and the fixation of carbon, and when vegetable matters are used, as they constantly are, as the food of animals, the latter exercise another force in the act of assimilation. Again, it has been found that certain organic products have already been prepared artificially by chemical processes, and it is not illogical or unreasonable to hope that even such substances as gelatine and sugar may be formed in a similar manner. But $\mathrm{Mr}$. Baker also draws distinctions between the powers of life and those of inorganic matter; for instance, no chemical process can form a cell, and no machine of human invention possesses the power of self-development or of generation. The whole tendency of Mr. Baker's paper appears to be to show that the forces excited in living bodies are analogous to other forces, such as those denominated galvanic, chemical, \&c., and that the belief in the doctrine of the mutual convertibility of all forces, vital and physical, and their unity and imperishability, is by no means inconsistent with a belief in the existence of an allpowerful Creator.

V. An illustration of extensive Cancer, traceable to dispersion from the primary Tumour, as distinguished from its constitutional reproduction. By Charles H. Moore.-After describing the different modes in which cancer is disseminated, Mr. Moore relates the case which forms the subject of his paper, and in which the communication between the cancer and the surrounding parts was maintained by the agency of a creamy liquid, and the continuity of distant tissues with the original disease was maintained.

The patient was a woman, aged 48 , who suffered from a rapidly-increasing tumour of the left breast, which soon formed

84-XLII. 
an ulcer. There was a cluster of enlarged and moveable glands in the axilla, and a firm and globular gland was felt above the clavicle and behind the sterno-mastoid muscle. An operation was performed with the view of relieving local suffering and temporarily arresting the growth of the tumour, and the knife was employed in the usual way, care being taken to remove with the tumour all the thickened integument as well as the diseased axillary glands. In about two months and a half the wound was entirely healed, but two small, hard nodules were perceived below the cicatrix; and in about two months more six tumours were observable under the skin below the scar. But Mr. Moore specially draws attention to the fact that above the scar the integuments were pale, flat, and supple, and the supra-clavicular gland behind the sterno-mastoïd was not larger than before the operation. The disease again spread rapidly in all directions, but the contrast of the appearances above and below the scar was still very striking, the tumefaction and discoloration being much more marked below than above. Death ensued in a few months more, and it was then found that the body was not emaciated, and the muscles were well nourished. The diseased mass presented a creamy whiteness, and the surface, when cut or squeezed, yielded abundantly a perfectly white juice resembling cream. The disease was found upon microscopical examination to be cancerous, and the milky or creamy fluid contained numerous oil-globules. From the examination of the organs affected with the disease, Mr. Moore infers that after the operation was performed, and a tough, transverse scar was established across the left side of the chest, the lymphatic current was temporarily arrested in its course upwards, but was conducted downwards into the lower part of the left side of the thorax, and subsequently. to the bronchial glands, diaphragm and liver, which last, however, presented only one cancerous nodule. The mesenteric glands were not affected, and hence the patient was maintained in a state of general good nutrition. Mr. Moore considers that in this case the disease was nourished from healthy blood, and that the whole source of the cancerous impregnation was derived from the left mammary region, whence alone the oily products overflowed the tissues.

VI. On the Examination of Patients suffering from Deafness. By Thomas Smith.-In this paper Mr. Thomas Smith, who disclaims the character of a specialist in diseases of the ear, describes generally the affections to which that organ is liable, and the methods by which they are most readily investigated. He draws a comparison between the eye and the ear to prove the essential difficulty attendant upon the investigation of dis- 
eases of the latter as compared with the former, the one organ presenting a number of transparent structures which are easily seen, and the other having nearly all its structures concealed in bony cavities. Mr. Smith describes the instruments necessary for investigating diseases of the ear, and shows that they need be only few in number and simple in construction, and he gives directions as to the inquiries which should be made of the patient when examining into the history of the disease. The use of an ordinary watch is available for ascertaining generally the existence and the degree of deafness; but in exploring the condition of the external meatus, a small concave mirror may be used, together with the simple tubular speculum of Sir W. Wilde. To ascertain the patency of the Eustachian tube, the patient should blow air into the tympanum while the surgeon listens, by means of the otoscope, to the effect produced. The Eustachian catheter is also described by Mr. Smith, and also the mode of introducing it, which is a matter of some difficulty to be overcome only by practice upon the dead subject, or on an anatomical preparation.

VII. On Disease of the Mitral Valve. By J. Annew, M.D. -Dr. Andrew commences his paper by relating a case in which it would appear that a disease of the mitral valve was cured. The patient was a girl, aged 9 , in whom the physical signs and some of the rational symptoms very clearly indicated mitral regurgitation, but after treatment for some months the physical and other signs began to improve, and eventually, but not until about three years from the date of her first attendance, the characteristic murmur disappeared, and she became quite well. The improvement was much greater than in any other that Dr. Andrew has ever observed, but he has notes of several cases in which the progress made was so considerable that he hopes the results in the present case may be equally favorable. In considering the question of the possibility of recovering from mitral disease, it is necessary to distinguish between the several forms of lesion which give rise to regurgitation, for some of these appear to be remediable; but of mitral constriction Dr. Andrew says nothing, believing it to be all but a hopeless affection. Incompetence, however, depends upon causes which are sometimes removable. In rheumatic fever, for instance, it may happen that only the surface of the valve is inflamed, and the murmur may disappear, and no trace of the disease will be left, but in other cases the substance of the valve is inflamed, and the murmur is developed more slowly, and the disease is far more permanent. Supposing that mitral disease is remediable in certain cases, Dr. Andrew suggests that the curative 
measures should have for their object to diminish the sum-total of the blood in the body; to maintain the nutrition of the heart and its muscular power, and to diminish the frequency and energy of the heart's action. The first object is promoted by prescribing a somewhat restricted diet; the second by the employment of some preparation of iron,-preferably the tincture of the perchloride, - or quinine; and the third by the avoidance of all excitement and by the use of digitalis.

VIII. Report on the Cases of Cholera treated in the Wards of St. Bartholomew's Hospital during the epidemic of 1866. By William Church, M.B.-The number of cases admitted into the cholera wards of the hospital in the epidemic of 1866 was 136, being a smaller number than in former epidemics. Mr. Church arranges in tables the history of the cases admitted, giving the names, ages, and previous residences of the patients, together with the nature of the premonitory symptoms, if any existed, the date of death or of discharge, and other particulars. The proportion of deaths to admissions was 33.08 per cent., being a higher proportion than in the epidemic of 1854, but a lower one than in that of 1848 . Premonitory symptoms, by which Mr. Church means those which existed at least twelve hours before the commencement of the algide state, were absent in 43.1 per cent. of fatal cases, and in 21.6 per cent. of nonfatal cases. The temperature was noted in thirty-one cases, twenty-two of which were fatal, and nine recovered. As a result of his observations, Mr. Church considers that the fall of temperature below $94.5^{\circ}$ was an indication that the case was severe, and the chance of recovery small. The microscopical examination of the discharges did not lead, apparently, to any very important results, the chief microscopical characters of the stools being the presence of large cellular bodies resembling mucous corpuscles, and a quantity of faintly granular material, with numerous bodies resembling free nucleoli. The stools were sometimes of a brickdusty appearance, due in many instances to the presence of blood-corpuscles; and this appearance seemed to be invariably a fatal symptom. The treatment was of the most varied character, but it was uniformly unsatisfactory. In only one case, under Mr. Church's care, was the injection of saline fluid into the veins attempted. The measure was attended, apparently, with some success; but the patient died soon after the injection. In eighteen cases a post-mortem examination was made, and the appearances observed are arranged in a tabular form.

IX. Note on Dr. Roberts's method of estimating Diabetic 
Sugar. By Philip J. Hensley, M.A., M.B.-Dr. Roberts's rule for estimating the amount of sugar in a specimen of diabetic urine is to ferment the urine by means of yeast, having first taken the specific gravity, and then, in twenty-four hours, taking the specific gravity again, when the fermentation has ceased, and the scum has subsided. The density after fermentation is subtracted from the density before fermentation, when the "density lost" is ascertained, and the number of degrees of "density lost" indicates as many grains of sugar per fluid ounce. This method is virtually to estimate the amount of sugar by determining the weight of carbonic acid lost, instead of by actually collecting and measuring the carbonic acid, as is usually done. Mr. Hensley points out that the plan pursued by Dr. Roberts is open to several objections, and he shows, by a series of mathematical formulæ, the errors which it involves, but which, we may remark, are not very great, and do not much invalidate Dr. Roberts's rule, which, as Mr. Hensley admits, is extremely useful for ordinary purposes.

X. Observations on the Passage of certain Substances into the Urine in Healthy and Diseased States of the Kidneys. By Dyce Duckworth, M.D.-Dr. Duckworth, in this paper, embodies a series of experiments conducted by him during the last two years. The first series of experiments refers to the excretion of some pigmentary and odorous substances by the healthy kidney, including the iodide of ethyl, iodide of potassium, bromide of potassium, indigo, aniline, logwood, turmeric. and santonine. It is known that most of these substances can be detected in the urine when the kidneys are healthy, but in opposition to assertions to the contrary, Dr. Duckworth shows that the pigments of indigo and logwood are excreted by the healthy kidneys. The second series of experiments was made upon patients suffering from disease of the kidneys, and the question was, to determine whether medicinal and odoriferous substances passed through the unhealthy as well as the healthy organs. The general opinion was that they did not; but Dr. Duckworth shows that this opinion is not altogether supported by facts. In his examinations he employed chiefly santonine, iodide of potassium, and turpentine; and he found that, in a certain number of cases, where the patients were suffering from diseased kidneys, the above-mentioned substances were found in the urine. Santonine, Dr. Duckworth believes, has not been previously employed in the manner described in his experiments; but he observes that it is easily exhibited, is the least unpleasant of all the substances he has used, and also yields its reaction in a very marked manner. 
XI. Ligature of the External Iliac Artery. By Augustin PRICHARD.-Although ligature of the external iliac artery is a rare operation, it happened that the two cases recorded by $\mathrm{Mr}$. Prichard occurred at the same period in the Bristol Infirmary. In the first case there was aneurysm in both thighs, and the femoral artery on the one side and the external iliac on the other were successively tied, though at an interval of four years. The first operation, which consisted in tying the femoral artery, was quite successful, and the recovery was rapid; but the second was attended with considerable difficulty, owing to constitutional and other complications. The aneurysm in this latter case was immediately below Poupart's ligament, and evidently extended a little upwards, and the ligature of the external iliac was effected with some little difficulty, and was followed by local inflammation and great constitutional disturbance. But the ligature came away on the twenty-second day, and the patient seemed to be recovering, when he was seized with a fresh set of symptoms, partly neuralgic and partly inflammatory, and the limb was stiff, hard, and painful. Eventually, however, the man entirely recovered, and resumed his usual employment, which was that of a butcher. The second case, which occurred at the same time as the first, was one of femoral aneurysm, in which ligature of the external iliac was also performed; but in this instance the operation was performed without any difficulty, and the patient recovered slowly, and without any remarkably unfavorable complications.

XII. Remarks on all the Principal Cases of Injury of the Head admitted into the Hull General Infirmary during the Six Years from 1858 to 1863. By Charles Jewel Evans.-Mr. Jewel Evans in this paper arranges in a tabular form the history of forty cases of injury of the head, placing the simpler cases first, and afterwards the more serious and the fatal ones: but no cases of scalp-wound, unless complicated with serious injury, are admitted. More than half the cases were from falls into the hold of a vessel, or into a dry dock, and of the whole number more than half, namely twenty-two, ended fatally. In five instances the operation of trephining was employed, but the result was fatal in all but one, and $\mathrm{Mr}$. Evans agrees in the opinion now generally entertained that the operation of trephining is not one from which a hopeful result may be often expected. In many cases in which the trephine is used the injury is so serious that no favorable termination can be even hoped for, and many cases which recover without trephining would, in all probability, be seriously endangered by the operation. When recovery takes place after the use of the trephine, it is a great 
question whether the instrument deserves the credit of the cure. Venesection was employed in two cases both of which were fatal, and this operation is now comparatively little employed in injuries of the head, but Mr. Evans thinks that local bleeding, especially in mild cases, is sometimes decidedly attended with benefit. Mr. Evans confirms the general opinion that the discharge of thin watery fluid from the interior of the cranium, although rare, is pathognomonic of fracture of the base of the skull. Facial paralysis, on the same side as the injury, is quite as much pathognomonic of fracture of the base of the skull as discharge of serous fluid, and is even more pathognomonic of fracture of the petrous bone, the facial nerve being injured in its course through that bone. Very little reliance can be placed upon the state of the pupils in injuries of the head, but the most common condition is that of dilatation. The respiration, in the cases where it was noted, was generally slow and laboured, and the pulse was usually slow.

XIII. On the Value of the Thermometer as an Aid to the Physician. By W. Arsste Hollis, M.B.-The investigations which form the groundwork of this paper included an examination of more than sixty cases of phthisis, and a still larger number of other cases as diabetes, disease of the heart, aneurysm, typhoïd and typhus fevers, pneumonia, bronchitis, pleuritis, erysipelas, scarlatina, phlebitis, \&c., and Mr. Hollis establishes the following proposition from his researches, namely, that " all local disorders, whether organic or functional, have a tendency to modify the thermometric ranges of the body, either by producing local variations of temperature, or by affecting those of the system at large." He then draws up in two tables the causes which tend to lower, and those which tend to raise the temperature of the body. Among the causes leading to diminished temperature are evacuations of various kinds, as for instance, diarrhœa, and looseness of the bowels, but when this diarrhœe is caused by ulceration of the intestines, as in typhoïd fever, the temperature is not diminished, but is raised. Perspiration and hæmorrhage also tend to diminish the temperature, and the same effect is produced by blisters and other counter-irritants. It is stated that the favorable action of blisters in acute rheumatism may be explained in this view. Deficient aëration of the blood, from whatever cause, will also diminish the temperature of the body. On the other hand, the temperature is raised in many acute and febrile diseases, but still the height of the thermometer in a case of fever is no criterion, per se, of the severity of the attack, for the temperature may be very high in a favorable case, and much lower 
than that point in a fatal one. The rapid degeneration or destruction of tissue such as occurs in certain inflammations, and in tuberculosis, carcinoma, and ulceration, also tends to increase the temperature. On the whole, Mr. Hollis arrives at the conclusion that thermometric registrations, although valuable in themselves, do not supersede or lessen the value of careful observations of the general symptoms of disease, and that the real value to be assigned to any given temperature must depend upon a due consideration of the processes which have caused it.

XIV. Extraordinary anomalous Affection of the Nervous System in a Boy. By Luther Holden.-The subject of this curious case is a boy, a patient in the hospital, between twelve and thirteen years old, whose only morbid affection appears to be a small swelling of the neck about the size of a hen's egg, situated on the right side of the neck, and who usually seems to enjoy very good health. But when this swelling is touched, however slightly, a series of extraordinary phenomena immediately present themselves, the boy becomes deaf, dumb, and blind, insensible to pain, and tetanic. After remaining in this state some forty or fifty seconds, he recovers after drawing a deep sigh. The physiological and pathological nature of this affection has been investigated hitherto in vain, and all therapeutical appliances have been fruitless. A proposition has been made to remove the tumour, which seems to be of a fatty nature, but there is a division of opinion as to the probable result of such a proceeding. Mr. Holden's own opinion is that its removal would not be attended with any serious results, but the further history of the case is promised in a future number of the 'Reports.'

XV. Surgical Cases in the Devon and Exeter Hospital. By Philip Chinwell Delagarde.-This paper contains the record of thirty cases taken from the surgical practice of the hospital, and includes surgical affections of the hand and of the head and neck, together with several cases of cancer. In a note on scirrhus and other forms of cancer, Mr. Delagarde expresses his opinion in favour of operating in certain cases of this disease, as he inclines to the belief that cancer commences as a local affection and afterwards affects distant parts, in contradistinction to the circumstance that the local phenomena of scrofula and syphilis are manifestations resulting from constitutional taint. Even when little hope of an absolute cure can be entertained in cancer of the breast, Mr. Delagarde does not refuse to operate, because such a proceeding alleviates the symptoms of the disease and does not involve any risk to life in well-selected cases. The instances in which Mr. Delagarde 
thinks an operation advisable are those of scirrhus and epithelioma, but in encephaloïd cancer there is not much chance of cure by operation. He prefers the knife to the use of caustic pastes, and he is unacquainted with the effect of acid injections either in primary or secondary scirrhus.

XVI. On Tracheotomy in Children; its Method, its Dangers, and its Difficulties. By F. Howard Marsh.-In this paper Mr. Marsh records the conclusions at which he has arrived chiefly from his experience as House Surgeon at the Hospital for Sick Children. In the first place, he combats the objections which have sometimes been made against the operation, and he concludes that it can be as deliberately performed as any other surgical proceeding, that the struggles of the patient may be obviated by chloroform, and that the apprehension of dangerous hæmorrhage has been exaggerated. After a brief but careful description of the structures concerned in the operations for opening the windpipe, he draws a comparison between laryngotomy and tracheotomy, declaring his opinion to be in favour of the latter as being on the whole more efficacious, although laryngotomy is most easily performed. But laryngotomy affords but little space for the introduction of the tube, it injures the integrity of the larynx and the vocal apparatus, and the tube causes great irritation and distress. In tracheotomy, few structures of any very great importance are involved, the tube is easily introduced, and the risk of hæmorrhage is but slight. Mr. Marsh then points out the spot most suitable for the operation, which should not be too low down on the one hand, where the trachea is very deep seated, nor too high, where the thyroid gland is placed, and which it is expedient not to wound. The tube to be employed is then described, and the different steps of the operation are minutely related, together with the after-treatment, so far as surgical ministrations are concerned. Some cases are then recorded in which the operation was performed, and in some the proceeding was successful; in others, where the result was less fortunate, the causes of the failure are pointed out. The patient may die from the constitutional disease for the relief of which the operation was performed, or from the local effects of the operation itself, and sometimes in cases which promise well, some accidental circumstance produces a fatal result, as where a portion of false membrane becomes entangled in the canula. Ulceration about the wound, or of the trachea around the canula, is also a more common result and a more frequent source of danger than is generally supposed, and Mr. Marsh has notes of nine cases where this complication occurred. 
XVII. Case of Anchylosis of the Atlas to the Occipital Bone and of Dislocation and subsequent Anchylosis of the Atlas and Axis. By Professor Turner.-The case was taken from a specimen in the Anatomical Museum of the University of Edinburgh, but nothing was known of the history during life. From the appearances observed it seemed that the articular surfaces of the bones had been diseased in the first instance, the ligaments relaxed and destroyed, and the bones subsequently displaced and finally anchylosed. The size of the spinal canal was diminished in the locality where the disease existed, and it is inferred that the spinal cord must have been considerably atrophied or much compressed in the antero-posterior direction.

XVIII. A Discussion of the Mechanical Theories which have been advanced to account for the origin of Pulmonary Emphysema. By Philip J. Hensley, M.A., M.B.-Mr. Hensley first considers three modes of explanation which have been adopted in accounting for the existence of emphysema. One offered by Laennec supposed that the lesion was caused by obstruction of the bronchial tubes, and by the action of the inspiratory muscles, which, being more powerful than the expiratory, would force in more air than expiration was able to eliminate, and thus the air would become imprisoned. The second explanation attributes emphysema to violent efforts in coughing and other expiratory acts, which are supposed to cause rupture of the air vesicles. A third view is that pulmonary emphysema is " a secondary mechanical lesion depending upon some condition of the respiratory apparatus which leads to partially diminished bulk of the pulmonary tissue, and consequently disturbs the balance of the air in inspiration." To all these views Mr. Hensley takes exception, and although he is not himself prepared to offer any very satisfactory explanation of the cause of emphysema, he explains some circumstances which he thinks may give rise to stretching of the lung tissue, and thus cause dilatation of the vesicles. In almost all cases emphysema is preceded by frequent attacks of bronchitis, which disease prevents the free passage of air into the bronchi; and $\mathrm{Mr}$. Hensley thinks that if the bronchi leading to some parts of the lungs are impassably blocked up, the tension in inspiration will be borne unduly by neighbouring portions, and thus dilatation of some of the vesicles be established. But he regards the stretching of the lung tissue only as an accessory to the lesion, and he believes that there is in the first instance a degenerative process in the tissues as well as an inflammatory condition of the bronchial mucous membrane, and that emphy- 
sema is caused therefore not only by mechanical, but also by vital causes.

XIX. Respecting the Treatment of Fractures of the Lower Extremities in the Wards under the care of Mr. Paget. By J. Astley Bloxam.-Before describing the new forms of apparatus employed at St. Bartholomew's in the treatment of fractures of the lower extremities, Mr. Bloxam alludes to the circumstance that no apparatus is now employed in fractures of the femur in children and in fractures of the patella. It is found that splints, bandages, and other such applications, are attended with great inconvenience in young children, and that the fractures may be safely treated by rest and the maintenance of a suitable position of the limb. Fractures of the patella are also treated without any apparatus, the patient being confined to bed with the injured limb extended on a level, with sandbags placed on each side, and with a cradle to keep off the weight of the bed-clothes from the limb. In a few days from the time of the accident, the extensors cease to act, and the fragments approximate themselves, the union, however, being usually ligamentous. The description of the apparatus employed in the treatment of other fractures of the lower extremities is illustrated by diagrams, without the aid of which a description would be impossible. It should be mentioned, however, that in fractures of the thigh, the inconveniences of the long splint are obviated by some novel contrivances, and that the new apparatus employed in these cases is both useful and convenient.

XX. Case in which a large Hydatid Cyst was removed from the Chest, with ultimate complete recovery. By $\mathrm{R}_{\mathrm{Eg}} \mathrm{fNald}$ Southey, M.D.-The subject of this interesting case was a female, aged thirty-one, who suffered from a variety of anomalous symptoms, somewhat resembling neuralgia and hysteria, from cough, though without expectoration, pain in the right side of the chest, and dyspepsia. No benefit followed from the remedial measures adopted, and change of air seemed to make her worse than before. The local indications and the physical signs were very obscure, but at last a marked bulging made its appearance upon the right side of the spine, extending into the interscapular region. An exploratory incision was then made by means of a trocar, and a large quantity of puriform serous fluid was evacuated, and some relief was afforded. But eventually a free incision was made between the sixth and seventh ribs, and a soft jelly-like mass protruded which was recognised as part of an hydatid cyst, which was fortunately entirely removed, its 
size being about that of a pig's bladder, and it was perfectly unattached. It appeared to be formed in the sac of the pleura above the diaphragm, and was wholly unconnected with the liver. The patient was restored to health in less than three months after the operation, and although she suffered from occasional pain in the right side, and the lung on that side was probably permanently altered in structure, she appeared stouter and better looking, and actually increased in weight more than a stone.

XXI. Some Cases of Disease of the Brain. By JoHN Crocketr Fish, M.B. This paper contains a brief history of five cases of cerebral disease, three being cases of tubercular meningitis, one of acute meningitis, and one of abscess of the cerebellum. All the cases were fatal, and in all there was considerable difficulty in the diagnosis, owing to the anomalous nature of the symptoms. In the last case, where disease of the cerebellum existed, there was also disease of the petrous portion of the temporal bone, and it is remarked that the danger in cases of chronic otorrhœa (which had been the first symptom in the case), may be staved off almost indefinitely by attempts to solicit the return of the discharge, and by other appropriate means.

XXII. On Senile Scrofula. By James PAGet, F.R.S.Mr. Paget remarks that scrofula is much more common in advanced life than is generally supposed, and that instances of the kind are to be found with equal frequency in private and hospital practice. The diagnosis between scrofula and gout in the old is often difficult, and it must be made by observing the co-existing constitutional characters, or other marks of the disease; and in distinguishing scrofula from cancer in advanced life, it is to be remembered that the hard cancer is more common in the old than the young, and that cancer is painful and increases rapidly.

XXIII. The Anatomy of Brain Shocks. Part I. By George W. Calmender.-This paper, which is the first of a series, contains forty-eight cases of injury or disease of the brain. The whole series is to include a hundred cases of the same kind, all fatal, which occurred and were recorded by the late Dr. Kirkes and by Mr. Callender, during the years 18491859 inclusive. Before describing the series of fatal cases, Mr. Callender refers to a few instances of concussion or contusion of the brain, which show that various subjects are affected in a very different manner by these accidents, partly 
from the varying nature of the injury, but in some measure by the constitutional peculiarities of the individual. The fatal cases are arranged in three distinct groups, the first containing those which were fatal from pressure on the surface of the brain or the walls of the ventricles; the second containing cases in which portions of the brain were destroyed by disease; and the third comprising cases in which there was bleeding into various parts of the substance of the brain.

XXIV. A Case of Traumatic Aneurysm. By Holmes Coоте.- In this case there was a wound of the femoral artery and vein, with effusion of blood into the limb. The case seemed to be doing well under pressure, but during the treatment the patient became excited in conversation, and from this and other causes, profuse hæmorrhage occurred, and although the limb was promptly amputated a fatal result ensued.

The reports conclude with an extract from the "Statistical Report of the Hospital for the year 1866."

\section{Review IV.}

\section{Structure and Function of the Retina.}

THE microscopic examination of the retina is beset with such formidable difficulties that even those who are most conversant with the subject scarcely succeed in mastering all its details, or in reconciling their own observations with the conflicting statements of others. And since the interest which attaches to researches of this kind is seldom felt beyond the circle of those personally engaged in them, it cannot be expected that the medical practitioner already overtasked by the demands of a many-sided science should follow very closely the laborious advance of retinal anatomy.

But looking beyond this drudgery of anatomical detail to the fair prospect which it offers of a final explanation of the phenomena of retinal vision; looking also to the extreme interest and importance of the scientific problems therein involved, we cannot but regard the numerous contributions recently made to retinal anatomy with a satisfaction proportionate to their present and prospective significance; for whether we measure their value by the amount of time and pains bestowed on them, or by their own proper merits, we have equal reason to con- 